\title{
A Diamond Principle Consistent with AD
}

\author{
Daniel Cunningham
}

\begin{abstract}
We present a diamond principle $\searrow_{\mathbb{R}}$ concerning all subsets of $\Theta$, the supremum of the ordinals that are the surjective image of $\mathbb{R}$. We prove that $\searrow_{\mathbb{R}}$ holds in Steel's core model $\mathbf{K}(\mathbb{R})$, a canonical inner model for determinacy.
\end{abstract}

\section{Introduction}

Jensen [5, p. 293] formulated his diamond principle $\nabla_{\kappa}$ and proved that this principle holds in $L$, the class of constructible sets. The assertion $\nabla_{\kappa}$ is a combinatorial property about all subsets of a regular cardinal $\kappa>\omega$.

Definition 1.1 Let $\kappa$ be a regular uncountable cardinal. Then $\nabla_{\kappa}$ is the statement that there is a sequence $\left\langle A_{\alpha}: \alpha \in \kappa\right\rangle$ such that $A_{\alpha} \subseteq \alpha$ for $\alpha \in \kappa$, and for all $A \subseteq \kappa$, the set

$$
\left\{\alpha \in \kappa: A \cap \alpha=A_{\alpha}\right\}
$$

is stationary in $\kappa$.

When $\kappa=\omega_{1}$, the diamond principle $\nabla_{\omega_{1}}$ is denoted by $\diamond$. Clearly $\diamond$ implies that the set of reals $\mathbb{R}$ can be well-ordered in order type $\omega_{1}$, as every subset of $\omega$ must appear among the $A_{\alpha}$ 's. Consequently, the principle $\diamond$ is inconsistent with the axiom of determinacy $(\mathrm{AD})$.

Remark 1.2 The combinatorial principles $\diamond$ and $\nabla_{\kappa}$ both hold in $L$ (see Devlin [3]) and in the Steel core model K (see Steel [11] and Schimmerling [9]). For any real $x$, the inner models $L[x]$ and $\mathbf{K}[x]$ are defined by allowing sets to be constructed relative to the real $x$ (see [3] and Hauser [4]). One can show that $\diamond$ and $\nabla_{\kappa}$ hold in $L[x]$ and $\mathbf{K}[x]$ as well.

Received June 19, 2012; accepted January 5, 2015

First published online April 21, 2017

2010 Mathematics Subject Classification: Primary 03E15; Secondary 03E45, 03 E60

Keywords: diamond principles, determinacy, Steel's core model $\mathbf{K}(\mathbb{R})$

(C) 2017 by University of Notre Dame 10.1215/00294527-2017-0008 
Given a regular uncountable cardinal $\kappa$, we see that $\nabla_{\kappa}$ also implies that there is a well-ordering of $\mathbb{R}$. Of course, any diamond principle that induces a well-ordering of the reals is inconsistent with AD.

Let $\Theta$ be the supremum of the ordinals which are the surjective image of $\mathbb{R}$. In this article we introduce a combinatorial principle $\nabla_{\mathbb{R}}$ which generalizes $\nabla_{\Theta}$. In addition, we prove that $\diamond_{\mathbb{R}}$ holds in $\mathbf{K}(\mathbb{R})$, an inner model where one can assume AD.

Many of our proofs apply forcing arguments which can be easily understood by anyone who is familiar with the basics of forcing as presented, say, in Kunen [7]. Moreover, our proofs require no consequences of the axiom of choice (AC) that are not available in $\mathbf{K}(\mathbb{R})$.

Recall that to prove the Löwenheim-Skolem theorem, one applies AC to show that every countable satisfiable theory has a countable model. We now identify a weaker form of this theorem that can be proved in ZF. Let $\bigwedge \Psi$ be the conjunction of a finite set $\Psi$ of sentences of set theory. If $\bigwedge \Psi$ has a transitive model (e.g., $V_{\alpha}$ ), then Barwise [1, Theorem 8.10] proves in ZF that $\bigwedge \Psi$ has a transitive model in $L$. Therefore, a version of the Löwenheim-Skolem theorem is provable without AC, namely,

$$
\mathrm{ZF} \vdash \text { (" } \Psi \text { has a transitive model” } \Longrightarrow \text { “ } \Psi \text { has a countable transitive model”). }
$$

Hence, if a sentence $\varphi$ is true in every countable transitive model of a given finite fragment of ZF, then it follows (using the reflection principle) that $\varphi$ holds. Since this semantical argument can be formalized in ZF, it thus follows that ZF $\vdash \varphi$.

Does a forcing argument yield a proof in ZF? There will be times when we want to prove that a certain statement $\varphi$ holds in $V$, the universe of sets. To do this, we may implement the following forcing argument: We naively presume that $V$ is a countable transitive model of ZF and then construct a generic extension $V[G]$. After working in $V[G]$, we will conclude that $\varphi$ holds in $V$. Such a forcing argument shows that there exists a proof of $\varphi$ in $\mathrm{ZF}$ because it will allow us to conclude that $\varphi$ is true in every countable transitive model of a particular finite fragment of ZF. To see this, let $\Psi$ be a finite set of axioms in ZF so that whenever $V$ is a countable transitive model of $\Psi$, we can be assured that $V$ and $V[G]$ will satisfy the specific axioms of ZF that we used in our forcing argument. Hence, $\varphi$ must be true in $V$ and thus, as noted in the previous paragraph, $\mathrm{ZF} \vdash \varphi$.

\section{A Brief Overview of $K(\mathbb{R})$}

We summarize the fundamental notions which will be assumed in the remaining sections of the article. Let $\omega$ be the set of all natural numbers. Then $\mathbb{R}={ }^{\omega} \omega$ is the set of all functions from $\omega$ to $\omega$. We call $\mathbb{R}$ the set of reals.

Steel [10] defines a countably iterable $\mathbb{R}$-premouse which we will denote as $\mathcal{M}$. Each premouse $\mathcal{M}$ is a model of $V=L(\vec{E}, \mathbb{R})$, where $\vec{E}$ is a fine extender sequence over $\mathbb{R}$. The inner model $\mathbf{K}(\mathbb{R})$ is then defined to be the "union" of these $\mathbb{R}$-premice. (Readers are directed to [10, Sections 1,2] for more details on the definition of $\mathbf{K}(\mathbb{R})$.) Since $\mathbf{K}(\mathbb{R})$ has a fine structure similar to that of $L(\mathbb{R})$, we have the following lemma.

Lemma 2.1 Assume $V=\mathbf{K}(\mathbb{R})$, and let $S$ be a set. Then there is a surjection $f: \lambda \times \mathbb{R} \rightarrow S$ for some ordinal $\lambda$. 
Definition 2.2 We let $\mathbb{Q}=(Q, \leq)$ be such that $Q=\left\{s \in{ }^{n} \mathbb{R}: n \in \omega\right\}$ and for $s, t \in Q$, define $s \leq t$ if and only if $\operatorname{dom}(s) \geq \operatorname{dom}(t)$ and $t=s \uparrow \operatorname{dom}(t)$.

We will write $s \in \mathbb{Q}$ to mean that $s \in Q$. We let $\mathbb{1}$ denote the largest element in the partial order $\mathbb{Q}$. Let $\mathbf{K}(\mathbb{R})^{\mathbb{Q}}$ represent the class of $\mathbb{Q}$-names in $\mathbf{K}(\mathbb{R})$, that is,

$$
\mathbf{K}(\mathbb{R})^{\mathbb{Q}}=\left\{\tau \in \mathbf{K}(\mathbb{R}):(\tau \text { is a } \mathbb{Q} \text {-name })^{\mathbf{K}}(\mathbb{R})\right\} .
$$

For each element $a \in \mathbf{K}(\mathbb{R})$, we define the $\mathbb{Q}$-name $\check{a}$ by the following recursion: $\check{a}=\{\langle\breve{b}, \mathbb{1}\rangle: b \in a\}$. By fusing the results of Cunningham [2] and [4], we have the following theorem.

Theorem 2.3 Let $G$ be $\mathbb{Q}$-generic over $\mathbf{K}(\mathbb{R})$. Then

(1) $\mathbf{K}(\mathbb{R})[G] \models \mathrm{ZFC}$,

(2) $\Theta^{\mathbf{K}(\mathbb{R})}=\omega_{1}^{\mathbf{K}(\mathbb{R})[G]}$.

In addition, the following statements are true in $\mathbf{K}(\mathbb{R})[G]$ :

(1) $\mathbb{R}^{\mathbf{K}(\mathbb{R})}$ and $G$ can be "canonically" coded by a single real $x$;

(2) $V=\mathbf{K}[x]$.

\section{The Closed Unbounded Filter on $\Theta$}

We will investigate, within $\mathbf{K}(\mathbb{R})$, the closed unbounded filter on $\Theta$. First, we recall the classic definitions and results concerning such filters on $\kappa$ whenever $\kappa$ is a regular uncountable cardinal.

(1) A set $X \subseteq \kappa$ contains its limit points less than $\kappa$ when the following holds: for each limit ordinal $\alpha<\kappa$, if $\sup (X \cap \alpha)=\alpha$, then $\alpha \in X$.

(2) A set $C \subseteq \kappa$ is closed unbounded in $\kappa$ if $C$ is an unbounded subset of $\kappa$ containing all its limit points less than $\kappa$. (This notion of a closed unbounded set is absolute.)

(3) A set $S \subseteq \kappa$ is stationary in $\kappa$ if $S \cap C \neq \varnothing$ whenever $C$ is closed unbounded in $\kappa$.

(4) The closed unbounded filter on $\kappa$ is the set $\mathcal{F}=\{X \subseteq \kappa: C \subseteq X$ for some closed unbounded subset $C$ of $\kappa\}$.

(5) $(\mathrm{ZF}+\mathrm{AC})$ The closed unbounded filter $\mathcal{F}$ on $\kappa$ is $\kappa$-complete; that is, for any $\lambda<\kappa$ and $\left\{X_{\alpha}: \alpha<\lambda\right\} \subseteq \mathcal{F}$ we have that $\bigcap_{\alpha<\lambda} X_{\alpha} \in \mathcal{F}$. Moreover, $\mathcal{F}$ is a normal filter; namely, $\mathscr{F}$ is closed under diagonal intersections.

In $\mathbf{K}(\mathbb{R})$ one can prove that $\Theta$ is a regular cardinal. When assuming $\mathrm{AD}$, one can also show that $\Theta$ is a regular limit cardinal in $\mathbf{K}(\mathbb{R})$. Let $\mathcal{F}$ be the closed unbounded filter on $\Theta$, that is,

$$
\mathcal{F}=\{X \subseteq \Theta: C \subseteq X \text { for some } C \text { that is closed unbounded in } \Theta\} .
$$

We will soon show that in $\mathbf{K}(\mathbb{R})$ one does not need $\mathrm{AC}$ to prove that $\mathcal{F}$ is a $\Theta$-complete normal filter and that $\mathcal{F}$ is $\mathbb{R}$-complete.

Theorem 3.1 Assume $V=\mathbf{K}(\mathbb{R})$. Suppose that $C_{x}$ is closed unbounded in $\Theta$ for each $x \in \mathbb{R}$. Then $\bigcap_{x \in \mathbb{R}} C_{x}$ is also closed unbounded in $\Theta$.

Proof Assume $V=\mathbf{K}(\mathbb{R})$. Let $\left\langle C_{x}: x \in \mathbb{R}\right\rangle$ be a sequence where each $C_{x}$ is closed unbounded in $\Theta$, and let $I=\bigcap_{x \in \mathbb{R}} C_{x}$. We will prove that $I$ is also closed 
unbounded in $\Theta$ through the use of a forcing argument. Let $G$ be $\mathbb{Q}$-generic over $\mathbf{K}(\mathbb{R})$. Thus, by absoluteness,

$$
\mathbf{K}(\mathbb{R})[G] \models\left(C_{x} \text { is closed unbounded in } \omega_{1}\right)
$$

for each $x \in \mathbb{R}^{\mathbf{K}}(\mathbb{R})$, the set of reals in the ground model. Since $\mathbb{R}^{\mathbf{K}(\mathbb{R})}$ is countable in $\mathbf{K}(\mathbb{R})[G]$, it follows that

$$
\mathbf{K}(\mathbb{R})[G] \models\left(I \text { is closed unbounded in } \omega_{1}\right) .
$$

Again, by absoluteness, we conclude that

$$
\mathbf{K}(\mathbb{R}) \models(I \text { is closed unbounded in } \Theta) .
$$

This completes the proof.

Corollary 3.2 Assume $V=\mathbf{K}(\mathbb{R})$. Suppose that $B_{x} \subseteq \Theta$ for each $x \in \mathbb{R}$. If $\bigcup_{x \in \mathbb{R}} B_{x}$ is stationary in $\Theta$, then for some $x \in \mathbb{R}$ the set $B_{x}$ is also stationary in $\Theta$.

Proof Assume $A=\bigcup_{x \in \mathbb{R}} B_{x}$ is stationary in $\Theta$. Suppose, for a contradiction, that each $B_{x}$ is not stationary. Let $C$ be the set of all closed unbounded subsets of $\Theta$. Lemma 2.1 implies (by minimizing an ordinal) that there is a function $F: \mathbb{R} \times \mathbb{R} \rightarrow \complement$ so that for each $x \in \mathbb{R}$ there is a $y \in \mathbb{R}$ such that $F(x, y) \cap B_{x}=\varnothing$. By Theorem 3.1, $C=\bigcap_{\langle x, y\rangle \in \mathbb{R} \times \mathbb{R}} F(x, y)$ is closed unbounded in $\Theta$. Since $A \cap C=\varnothing$, this contradicts the fact that $A$ is stationary.

Theorem 3.3 Assume $V=\mathbf{K}(\mathbb{R})$. The closed unbounded filter $\mathcal{F}$ on $\Theta$ is both $\Theta$-complete and closed under diagonal intersection.

Proof Let $\lambda<\Theta$, and let $\left\{X_{\alpha}: \alpha<\lambda\right\} \subseteq \mathcal{F}$. We will prove, without AC, that $\bigcap_{\alpha<\lambda} X_{\alpha} \in \mathcal{F}$. Let $\mathcal{C}$ be the set of all closed unbounded subsets of $\Theta$. Since we are working in $\mathbf{K}(\mathbb{R})$, Lemma 2.1 implies that there is a function $\Phi: \lambda \times \mathbb{R} \rightarrow \mathcal{C}$ such that, for each $\alpha<\lambda$, there is an $x \in \mathbb{R}$ such that $\Phi(\alpha, x) \subseteq X_{\alpha}$. By Theorem 3.1, each set $C_{\alpha}=\bigcap_{x \in \mathbb{R}} \Phi(\alpha, x)$ is closed unbounded in $\Theta$. Since $C_{\alpha} \subseteq X_{\alpha}$ for each $\alpha<\lambda$, it now follows that $\bigcap_{\alpha<\lambda} C_{\alpha} \subseteq \bigcap_{\alpha<\lambda} X_{\alpha}$, where $\bigcap_{\alpha<\lambda} C_{\alpha}$ is closed unbounded in $\Theta$. Hence, $\bigcap_{\alpha<\lambda} X_{\alpha} \in \mathcal{F}$. A similar argument will show that the filter $\mathcal{F}$ is closed under diagonal intersections.

Theorem 3.4 Assume $V=\mathbf{K}(\mathbb{R})$. The closed unbounded filter $\mathscr{F}$ on $\Theta$ is $\mathbb{R}$-complete; that is, for any $\left\{A_{x}: x \in \mathbb{R}\right\} \subseteq \mathcal{F}$ we have that $\bigcap_{x \in \mathbb{R}} A_{x} \in \mathscr{F}$.

Proof Let $\left\{A_{x}: x \in \mathbb{R}\right\} \subseteq \mathcal{F}$. We will prove, again without $\mathrm{AC}$, that $\bigcap_{x \in \mathbb{R}} A_{x} \in \mathcal{F}$. Let $\mathcal{C}$ be the set of all closed unbounded subsets of $\Theta$. Since we are working in $\mathbf{K}(\mathbb{R})$, Lemma 2.1 yields a function $\Phi: \mathbb{R} \rightarrow \zeta$ such that for each $x \in \mathbb{R}$ there is a $y \in \mathbb{R}$ such that $\Phi(y) \subseteq A_{x}$. By Theorem 3.1, the set $C=\bigcap_{y \in \mathbb{R}} \Phi(y)$ is closed unbounded in $\Theta$. Since $C \subseteq \bigcap_{x \in \mathbb{R}} A_{x}$, we have $\bigcap_{x \in \mathbb{R}} A_{x} \in \mathcal{F}$.

We now show that $\mathbb{Q}$-forcing over $\mathbf{K}(\mathbb{R})$ preserves stationary sets (see Kanamori [6, Lemma 10.14]).

Theorem 3.5 Let $G$ be $\mathbb{Q}$-generic over $\mathbf{K}(\mathbb{R})$. Suppose that

$$
\mathbf{K}(\mathbb{R})[G] \models \text { "C is closed unbounded in } \omega_{1} \text {." }
$$

Then there is a $D \in \mathbf{K}(\mathbb{R})$ such that $D \subseteq C$ and

$$
\mathbf{K}(\mathbb{R}) \models \text { “D is closed unbounded in } \Theta \text {.” }
$$


Proof Let $p \in G$ be such that

$$
p \Vdash \text { " } \dot{C} \text { is closed unbounded in } \omega_{1}, "
$$

where $\dot{C}$ is a $\mathbb{Q}$-name for $C$. For each $\alpha \in \Theta$, define

$$
X_{\alpha}=\{\xi \in \Theta:(\exists q \leq p)[q \Vdash “ \breve{\xi} \text { is the least element in } \dot{C} \backslash(\check{\alpha}+1) \text { ” }\} .
$$

Since there is a mapping from $\mathbb{R}$ onto $\mathbb{Q}$, it follows that each $X_{\alpha}$ is bounded in $\Theta$. Let $f(\alpha)=\sup \left(X_{\alpha}\right)<\Theta$, for each $\alpha \in \Theta$. Now define

$$
D=\{\beta \in \Theta:(\forall \alpha<\beta)(f(\alpha)<\beta)\} .
$$

Since $D$ is the diagonal intersection of the sequence $\left\langle Y_{\alpha}: \alpha<\Theta\right\rangle$, where

$$
Y_{\alpha}=\{\beta \in \Theta: f(\alpha)<\beta\},
$$

we conclude that $D$ is closed unbounded in $\Theta$.

To show that $D \subseteq C$, it is sufficient to prove that $p \Vdash \check{D} \subseteq \dot{C}$. Suppose that $p \nVdash \check{D} \subseteq \dot{C}$. Thus, for some $q \leq p$ and $\beta \in D$, we have that $q \Vdash \check{\beta} \notin \dot{C}$. Let $H$ be $\mathbb{Q}$-generic over $\mathbf{K}(\mathbb{R})$ so that $q \in H$. Thus, in $\mathbf{K}(\mathbb{R})[H]$ we have that $\dot{C}_{H}$ is closed unbounded in $\omega_{1}$ and $\beta \notin \dot{C}_{H}$. Let $\alpha=\sup \left(\dot{C}_{H} \cap \beta\right)$. Since $\beta \notin \dot{C}_{H}$, we conclude that $\alpha<\beta$ and $\dot{C}_{H} \cap(\alpha, \beta]=\varnothing$, where $(\alpha, \beta]=\{\gamma: \alpha<\gamma \leq \beta\}$. Since $\beta \in D$ and $\alpha<\beta$, we have $(\star) f(\alpha)<\beta$. Because $\dot{C}_{H}$ is unbounded, there is a least $\xi$ such that $\xi \in \dot{C}_{H} \backslash(\alpha+1)$. It follows that $\xi \in X_{\alpha}$ and $\xi>\beta$. Hence, $\beta<\xi \leq \sup \left(X_{\alpha}\right)=f(\alpha)$. Therefore, $\beta<f(\alpha)$ which contradicts $(\star)$.

\section{Corollary 3.6 Let $G$ be $\mathbb{Q}$-generic over $\mathbf{K}(\mathbb{R})$. Suppose that}

$$
\mathbf{K}(\mathbb{R}) \models \text { "S is stationary in } \Theta \text {." }
$$

Then $\mathbf{K}(\mathbb{R})[G] \models$ "S is stationary in $\omega_{1}$."

\section{Diamond Principles in $\mathrm{K}(\mathbb{R})$}

Any sequence of the form $\left\langle\mathcal{A}_{\alpha, x}:\langle\alpha, x\rangle \in \Theta \times \mathbb{R}\right\rangle$ will be referred to as an $\mathbb{R}$-sequence. The following principle $\nabla_{\mathbb{R}}$ asserts the existence of an $\mathbb{R}$-sequence with some special combinatorial properties concerning all the subsets of $\Theta$.

Definition 4.1 The diamond principle $\diamond_{\mathbb{R}}$ is the statement: there is a sequence $\left\langle\mathcal{A}_{\alpha, x}:\langle\alpha, x\rangle \in \Theta \times \mathbb{R}\right\rangle$ such that $\mathcal{A}_{\alpha, x} \subseteq \alpha$ for each $\langle\alpha, x\rangle \in \Theta \times \mathbb{R}$ and for every $A \subseteq \Theta$ there exists an $x \in \mathbb{R}$ so that the set

$$
\left\{\alpha \in \Theta: A \cap \alpha=\mathcal{A}_{\alpha, x}\right\}
$$

is stationary in $\Theta$. We will call $\left\langle\mathcal{A}_{\alpha, x}:\langle\alpha, x\rangle \in \Theta \times \mathbb{R}\right\rangle$ a $\mho_{\mathbb{R}}$-sequence.

We will now show that $\diamond_{\mathbb{R}}$ holds in $\mathbf{K}(\mathbb{R})$.

Theorem 4.2 Assume $V=\mathbf{K}(\mathbb{R})$. Then there is a $\searrow_{\mathbb{R}}$-sequence.

Proof Let $\Theta=\Theta^{\mathbf{K}(\mathbb{R})}$, and let $\mathbb{Q}$ be as in Definition 2.2. Theorem 2.3 states that $\Theta=\omega_{1}^{\mathbf{K}(\mathbb{R})[G]}$ for any $G$ which is $\mathbb{Q}$-generic over $\mathbf{K}(\mathbb{R})$.

Claim 1 We have that $\mathbf{K}(\mathbb{R}) \models \mathbb{1} \Vdash$ "there is a $\diamond$-sequence."

Proof of Claim $1 \quad$ Let $G$ be $\mathbb{Q}$-generic over $\mathbf{K}(\mathbb{R})$. By Theorem 2.3, we have that $\mathbf{K}(\mathbb{R})[G]=K[x]$ for a real $x$. It follows (see Remark 1.2) that there is a $\diamond$-sequence in $\mathbf{K}(\mathbb{R})[G]$. 
Let $\check{\Theta}$ be a canonical name for $\Theta$. Let $p \in \mathbb{Q}$ and $\delta \in \mathbf{K}(\mathbb{R})^{\mathbb{Q}}$ be such that

$$
p \Vdash(\delta \text { is a } \diamond \text {-sequence } \wedge \operatorname{dom}(\delta)=\check{\Theta}) \text {. }
$$

In $\mathbf{K}(\mathbb{R})$, let $\alpha \mapsto \tau_{\alpha}$ be a mapping from $\Theta$ to $\mathbf{K}(\mathbb{R})^{\mathbb{Q}}$ so that for each $\alpha \in \Theta$

$$
\left(\tau_{\alpha}\right)_{G}=\left(\delta_{G}\right)_{\alpha} \text { whenever } G \text { is } \mathbb{Q} \text {-generic over } \mathbf{K}(\mathbb{R}) \text { and } p \in G \text {. }
$$

Now define the sequence $\mathbb{D}=\left\langle\mathcal{A}_{\alpha, q}: \alpha \in \Theta \wedge q \leq p\right\rangle$, where

$$
\mathcal{A}_{\alpha, q}=\left\{\lambda \in \Theta: q \Vdash \check{\lambda} \in \tau_{\alpha}\right\}
$$

for $\alpha \in \Theta$ and $q \in \mathbb{Q}$ such that $q \leq p$. From (4.1) and (4.2) we conclude that $\mathcal{A}_{\alpha, q} \subseteq \alpha$ for all $\alpha \in \Theta$ and $q \leq p$. Clearly, $\mathbb{D} \in \mathbf{K}(\mathbb{R})$ and $\mathbb{D}$ can be viewed as an $\mathbb{R}$-sequence. We will prove that $\mathbb{D}$ is a $\diamond_{\mathbb{R}}$-sequence in $\mathbf{K}(\mathbb{R})$. Let $A \subseteq \Theta$ be an element in $\mathbf{K}(\mathbb{R})$.

Claim 2 There is a $q \leq p$ such that $\left\{\alpha \in \Theta: q \Vdash \check{A} \cap \check{\alpha}=\tau_{\alpha}\right\}$ is stationary in $\Theta$.

Proof of Claim 2 Suppose, for a contradiction, that for each $q \leq p$ the set

$$
\left\{\alpha \in \Theta: q \Vdash \check{A} \cap \check{\alpha}=\tau_{\alpha}\right\}
$$

is not stationary in $\Theta$. Let $\zeta$ be the set of all closed unbounded subsets of $\Theta$. Lemma 2.1 implies that there is a function $\Psi: \mathbb{Q} \times \mathbb{R} \rightarrow \zeta$ in $\mathbf{K}(\mathbb{R})$ such that, for all $q \leq p$, there is an $x \in \mathbb{R}$ such that

$$
\Psi(q, x) \cap\left\{\alpha \in \Theta: q \Vdash \check{A} \cap \check{\alpha}=\tau_{\alpha}\right\}=\varnothing .
$$

Let $I=\bigcap_{\langle q, x\rangle \in \mathbb{Q} \times \mathbb{R}} \Psi(q, x)$. By Theorem 3.1, I is closed unbounded in $\Theta$.

Now let $G$ be $\mathbb{Q}$-generic over $\mathbf{K}(\mathbb{R})$ so that $p \in G$. Thus, $\left(\omega_{1}=\check{\Theta}_{G}\right)^{\mathbf{K}(\mathbb{R})[G]}$. So, in $\mathbf{K}(\mathbb{R})[G]$ we have that $A$ is a subset of $\omega_{1}$ and $I$ is closed unbounded in $\omega_{1}$. By (4.1) we conclude that $\delta_{G}=\left\langle\mathcal{A}_{\alpha}: \alpha<\omega_{1}\right\rangle$ is a $\diamond$-sequence in $\mathbf{K}(\mathbb{R})[G]$. Therefore, the set

$$
\left\{\alpha<\omega_{1}: A \cap \alpha=\mathcal{A}_{\alpha}\right\}
$$

is stationary in $\mathbf{K}(\mathbb{R})[G]$, and hence, there is a $\beta \in I$ such that $A \cap \beta=\mathcal{A}_{\beta}$. So, in $\mathbf{K}(\mathbb{R})$, there is a $q \leq p$ such that $q \Vdash \check{A} \cap \check{\beta}=\tau_{\beta}$. Let $x \in \mathbb{R}$ be such that $\Psi(q, x) \cap\left\{\alpha \in \Theta: q \Vdash \check{A} \cap \check{\alpha}=\tau_{\alpha}\right\}=\varnothing$. Since $\beta \in I$, we have that $\beta \in \Psi(q, x)$. This contradiction shows that such a $q$ must exist.

Let $q \leq p$ be such that $S=\left\{\alpha \in \Theta: q \Vdash \check{A} \cap \check{\alpha}=\tau_{\alpha}\right\}$ is stationary.

Claim 3 We have that $A \cap \alpha=\mathcal{A}_{\alpha, q}$ for all $\alpha \in S$.

Proof of Claim $3 \quad$ Let $\alpha \in S$. Recall that $\mathcal{A}_{\alpha, q}=\left\{\lambda \in \Theta: q \Vdash \check{\lambda} \in \tau_{\alpha}\right\}$. Since $\alpha \in S$, we have that $q \Vdash \check{A} \cap \check{\alpha}=\tau_{\alpha}$. An elementary forcing argument will now show that $A \cap \alpha=\mathcal{A}_{\alpha, q}$.

Since $S \subseteq\left\{\alpha \in \Theta: A \cap \alpha=\mathcal{A}_{\alpha, q}\right\}$ and $S$ is stationary, we conclude that

$$
\left\{\alpha \in \Theta: A \cap \alpha=\mathcal{A}_{\alpha, q}\right\}
$$

is stationary in $\Theta$. Therefore, $\mathbb{D}$ is a $\searrow_{\mathbb{R}}$-sequence in $\mathbf{K}(\mathbb{R})$.

Our next theorem shows that the consistency of ZF + AD implies the consistency of $\mathrm{ZF}+\mathrm{AD}+\diamond_{\mathbb{R}}$ 
Theorem 4.3 Assume $V=L(\mathbb{R})$. Then $\diamond_{\mathbb{R}}$.

Proof The proof of Theorem 4.2 can easily be adapted to establish the result.

There are a number of generalizations of $\diamond$. We now identify one such generalization.

Definition 4.4 Let $S \subseteq \omega_{1}$. Then $\diamond(S)$ is the statement that there is a sequence $\left\langle\mathcal{A}_{\alpha}: \alpha \in S\right\rangle$ such that $\mathcal{A}_{\alpha} \subseteq \alpha$ for $\alpha \in S$ and, for all $A \subseteq \omega_{1}$, the set

$$
\left\{\alpha \in S: A \cap \alpha=\mathcal{A}_{\alpha}\right\}
$$

is stationary in $\omega_{1}$.

The diamond principle $\diamond(S)$ holds in $L$ and $\mathbf{K}$ when $S$ is stationary in $\omega_{1}$. Consider the following generalization of $\searrow_{\mathbb{R}}$.

Definition 4.5 Let $S \subseteq \Theta$. The diamond principle $\searrow_{\mathbb{R}}(S)$ is the statement: there exists a sequence $\left\langle\mathcal{A}_{\alpha, x}:\langle\alpha, x\rangle \in S \times \mathbb{R}\right\rangle$ such that $\mathcal{A}_{\alpha, x} \subseteq \alpha$ for $\langle\alpha, x\rangle \in S \times \mathbb{R}$ and, for every $A \subseteq \Theta$, there is an $x \in \mathbb{R}$ so that

$$
\left\{\alpha \in S: A \cap \alpha=\mathcal{A}_{\alpha, x}\right\}
$$

is stationary in $\Theta$. Such a sequence will be called a $\searrow_{\mathbb{R}}(S)$-sequence.

The proof of Theorem 4.2 can be modified, using Corollary 3.6, to establish our next theorem.

Theorem 4.6 Assume $V=\mathbf{K}(\mathbb{R})$. If $S$ is stationary in $\Theta$, then $a \diamond_{\mathbb{R}}(S)$-sequence exists.

In the statement $\nabla_{\mathbb{R}}(S)$ of Definition 4.5 , let us replace $\Theta$ with $\kappa$ and call this new statement $\nabla_{\kappa, \mathbb{R}}(S)$. The proofs of Corollary 3.6 and Theorem 4.2 can also be extended to prove the following theorem.

Theorem 4.7 Assume $V=\mathbf{K}(\mathbb{R})$. Let $\kappa \geq \Theta$ be a regular cardinal, and let $S$ be stationary in $\kappa$. Then there is a $\diamond_{\kappa, \mathbb{R}}(S)$-sequence.

We now note that $\searrow_{\mathbb{R}}$ yields another combinatorial principle which is the analogue of $\diamond^{\prime}$ (see [3, p. 127]).

Definition 4.8 Let $\mho_{\mathbb{R}}^{\prime}$ be the assertion: there exists a sequence $\left\langle\mathcal{A}_{\alpha}: \alpha \in \Theta\right\rangle$ such that

(1) $\mathcal{A}_{\alpha} \subseteq \mathcal{P}(\alpha)$ for each $\alpha \in \Theta$;

(2) for every $\alpha \in \Theta$ there is a surjection of $\mathbb{R}$ onto $\mathcal{A}_{\alpha}$;

(3) for every $A \subseteq \Theta$ the set $\left\{\alpha \in \Theta: A \cap \alpha \in \mathcal{A}_{\alpha}\right\}$ is stationary in $\Theta$.

Clearly, $\searrow_{\mathbb{R}}$ implies $\diamond_{\mathbb{R}}^{\prime}$. Thus, Theorem 4.2 implies the following result.

Theorem 4.9 Assume $V=\mathbf{K}(\mathbb{R})$. Then $\searrow_{\mathbb{R}}^{\prime}$ holds.

When $\kappa$ is an ordinal and $S \subseteq \kappa$, let $\vee_{\kappa, \mathbb{R}}^{\prime}(S)$ be the appropriate generalization of Definition 4.8. Theorem 4.7 clearly implies that $\mathbf{K}(\mathbb{R})$ also satisfies $\vee_{\kappa, \mathbb{R}}^{\prime}(S)$ when $S \subseteq \kappa$ is stationary and $\kappa \geq \Theta$ is regular.

We will present a generalization of $\searrow_{\mathbb{R}}^{\prime}$. Before doing this, we recall Jensen's principle $\diamond^{*}$. 
Definition 4.10 Let $\diamond^{*}$ be the statement: there exists a sequence $\left\langle\mathcal{A}_{\alpha}: \alpha \in \omega_{1}\right\rangle$ such that

(1) $\mathcal{A}_{\alpha} \subseteq \mathcal{P}(\alpha)$ for each $\alpha \in \omega_{1}$;

(2) for every $\alpha \in \omega_{1}$ there is a surjection of $\omega$ onto $\mathcal{A}_{\alpha}$;

(3) for every $A \subseteq \omega_{1}$ there is closed unbounded $C \subseteq \omega_{1}$ such that $A \cap \alpha \in \mathcal{A}_{\alpha}$ for all $\alpha \in C$.

Such a sequence will be called a $\diamond^{*}$-sequence.

The following generalization of $\searrow_{\mathbb{R}}^{\prime}$ corresponds to Jensen's principle $\diamond^{*}$.

Definition 4.11 Let $\diamond_{\mathbb{R}}^{*}$ be the assertion: there exists a sequence $\left\langle\mathcal{A}_{\alpha}: \alpha \in \Theta\right\rangle$ such that

(1) $\mathcal{A}_{\alpha} \subseteq \mathcal{P}(\alpha)$ for each $\alpha \in \Theta$;

(2) for every $\alpha \in \Theta$ there is a surjection of $\mathbb{R}$ onto $\mathcal{A}_{\alpha}$;

(3) for every $A \subseteq \Theta$ there is closed unbounded $C \subseteq \Theta$ such that $A \cap \alpha \in \mathcal{A}_{\alpha}$ for all $\alpha \in C$.

We will call $\left\langle\mathcal{A}_{\alpha}: \alpha \in \Theta\right\rangle$ a $\diamond_{\mathbb{R}}^{*}$-sequence.

In Definition 4.11, if we replace $\Theta$ with $\omega_{1}$ and $\mathbb{R}$ with $\omega$, then we obtain $\diamond^{*}$. The following lemma identifies two useful equivalences.

Lemma 4.12 Let $\left\langle\mathcal{A}_{\alpha}: \alpha \in \omega_{1}\right\rangle$ and $\left\langle\mathcal{A}_{\alpha}: \alpha \in \Theta\right\rangle$ be sequences. Then

(1) $\left\langle\mathcal{A}_{\alpha}: \alpha \in \omega_{1}\right\rangle$ is a $\diamond^{*}$-sequence if and only if $\left\langle\mathcal{A}_{\alpha}: \alpha \in S\right\rangle$ is a $\diamond^{\prime}(S)$-sequence for all stationary $S \subseteq \omega_{1}$.

(2) $\left\langle\mathcal{A}_{\alpha}: \alpha \in \Theta\right\rangle$ is a $\diamond_{\mathbb{R}}^{*}$-sequence if and only if $\left\langle\mathcal{A}_{\alpha}: \alpha \in S\right\rangle$ is a $\diamond_{\mathbb{R}}^{\prime}(S)$-sequence for all stationary $S \subseteq \Theta$.

In our next theorem we will prove that $\mathbf{K}(\mathbb{R}) \models \diamond_{\mathbb{R}}^{*}$. First we observe that, under $\mathrm{AC}$, the principle $\diamond^{*}$ can be restated as follows. There is a sequence $\left\langle\mathcal{A}_{\alpha, n}:\langle\alpha, n\rangle \in \omega_{1} \times \omega\right\rangle$ such that

(1) $\mathcal{A}_{\alpha, n} \subseteq \alpha$ for each $\alpha \in \omega_{1}$ and $n \in \omega$;

(2) for every $A \subseteq \omega_{1}$ there is closed unbounded $C \subseteq \omega_{1}$ so that for all $\alpha \in C$ there exists $n \in \omega$ such that $A \cap \alpha=\mathcal{A}_{\alpha, n}$.

We shall call such a sequence a ${ }^{*}$-sequence.

Theorem 4.13 Assume $V=\mathbf{K}(\mathbb{R})$. Then $\diamond_{\mathbb{R}}^{*}$ is true.

Proof Let $\Theta=\Theta^{\mathbf{K}}(\mathbb{R})$, and let $\mathbb{Q}$ be as in Definition 2.2. Theorem 2.3 states that $\Theta=\omega_{1}^{\mathbf{K}(\mathbb{R})[G]}$ for any $G$ which is $\mathbb{Q}$-generic over $\mathbf{K}(\mathbb{R})$.

Claim 1 We have that $\mathbf{K}(\mathbb{R}) \models \mathbb{1} \Vdash$ "there is a ${ }^{*}$-sequence."

Proof of Claim $1 \quad$ Let $G$ be $\mathbb{Q}$-generic over $\mathbf{K}(\mathbb{R})$. By Theorem 2.3, we have that $\mathbf{K}(\mathbb{R})[G]=\mathbf{K}[x]$ for some real $x$. Since $\mathbf{K}(\mathbb{R})[G] \models$ AC, it follows (see Remark 1.2) that there is a ${ }^{*}$-sequence in $\mathbf{K}(\mathbb{R})[G]$.

Let $\check{\Theta}$ and $\check{\omega}$ be canonical names for $\Theta$ and $\omega$, respectively. Let $p \in \mathbb{Q}$ and $\delta \in \mathbf{K}(\mathbb{R})^{\mathbb{Q}}$ be such that

$$
p \Vdash\left(\delta \text { is a }{ }^{*} \text {-sequence } \wedge \operatorname{dom}(\delta)=\check{\Theta} \times \check{\omega}\right) \text {. }
$$


In $\mathbf{K}(\mathbb{R})$, let $\langle\alpha, n\rangle \mapsto \tau_{\alpha, n}$ be a mapping from $\Theta \times \omega$ to $\mathbf{K}(\mathbb{R})^{\mathbb{Q}}$ so that for each $\alpha \in \Theta$ and $n \in \omega$,

$$
\left(\tau_{\alpha, n}\right)_{G}=\left(\delta_{G}\right)_{\alpha, n} \text { whenever } G \text { is } \mathbb{Q} \text {-generic over } \mathbf{K}(\mathbb{R}) \text { and } p \in G \text {. }
$$

Now for each $\alpha \in \Theta, n \in \omega$, and $q \leq p$, define the set

$$
B_{\alpha, n, q}=\left\{\lambda \in \Theta: q \Vdash \check{\lambda} \in \tau_{\alpha, n}\right\} .
$$

From (4.3) and (4.4), we conclude that $B_{\alpha, n, q} \subseteq \alpha$ whenever $\alpha \in \Theta, n \in \omega$, and $q \leq p$. For each $\alpha \in \Theta$, let $\mathcal{A}_{\alpha}=\left\{B_{\alpha, n, q}: n \in \omega\right.$ and $\left.q \leq p\right\}$. Clearly, $\left\langle\mathcal{A}_{\alpha}: \alpha \in \Theta\right\rangle \in \mathbf{K}(\mathbb{R})$. Using Lemma 4.12, we now prove that $\left\langle\mathcal{A}_{\alpha}: \alpha \in \Theta\right\rangle$

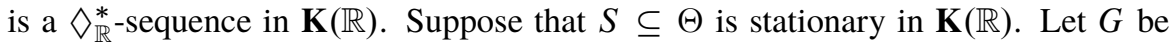
$\mathbb{Q}$-generic over $\mathbf{K}(\mathbb{R})$ so that $p \in G$. For each $\alpha \in \Theta$, define

$$
\mathbb{T}_{\alpha}^{G}=\left\{\left(\tau_{\alpha, n}\right)_{G}: n \in \omega\right\} .
$$

Consider the sequence $\left\langle\mathbb{T}_{\alpha}^{G}: \alpha \in \Theta\right\rangle$. Since $\Theta=\omega_{1}^{\mathbf{K}(\mathbb{R})[G]}$ and $p \in G$, it follows from (4.3) and (4.4) that $\left\langle\mathbb{T}_{\alpha}^{G}: \alpha \in \Theta\right\rangle$ is a $\diamond^{*}$-sequence in $\mathbf{K}(\mathbb{R})[G]$. Corollary 3.6 implies that $\mathbf{K}(\mathbb{R})[G] \models$ " $S \subseteq \omega_{1}$ is stationary." Thus, by Lemma 4.12(1), we have that $\left\langle\mathbb{T}_{\alpha}^{G}: \alpha \in S\right\rangle$ is a $\diamond^{\prime}(S)$-sequence in $\mathbf{K}(\mathbb{R})[G]$. So, for every such generic $G$, we have that $\left\langle\mathbb{T}_{\alpha}^{G}: \alpha \in S\right\rangle$ is a $\diamond^{\prime}(S)$-sequence in $\mathbf{K}(\mathbb{R})[G]$. The proof of Theorem 4.2 can now be modified to show that $\left\langle\mathcal{A}_{\alpha}: \alpha \in S\right\rangle$ is a $\mho_{\mathbb{R}}^{\prime}(S)$-sequence in $\mathbf{K}(\mathbb{R})$. Therefore, by Lemma 4.12(2), we conclude that $\left\langle\mathcal{A}_{\alpha}: \alpha \in \Theta\right\rangle$ is a $\mho_{\mathbb{R}}^{*}$-sequence in $\mathbf{K}(\mathbb{R})$.

The above proof of Theorem 4.13 easily generalizes to show that $\mathbf{K}(\mathbb{R})$ satisfies the corresponding principle $\diamond_{\kappa, \mathbb{R}}^{*}$ when $\kappa \geq \Theta$ is regular.

\section{A Second Proof of $\diamond_{\mathbb{R}}$}

Throughout this article, ZF has been our ambient theory. We now present a different proof of Theorem 4.2 assuming that $\mathbf{K}(\mathbb{R}) \models$ AD. The proof applies the coding lemma, an important result in descriptive set theory due to Moschovakis [8].

Since $\searrow_{\mathbb{R}}$ implies $\searrow_{\mathbb{R}}^{\prime}$, the following lemma shows that the principles $\searrow_{\mathbb{R}}^{\prime}$ and $\oslash_{\mathbb{R}}$ are equivalent in $\mathbf{K}(\mathbb{R})$. The proof of this lemma does not use AD.

Lemma 5.1 Assume $V=\mathbf{K}(\mathbb{R})$. Then $\diamond_{\mathbb{R}}^{\prime}$ implies $\diamond_{\mathbb{R}}$.

Proof Assuming $V=\mathbf{K}(\mathbb{R})$ and $\diamond_{\mathbb{R}}^{\prime}$, we will prove $\diamond_{\mathbb{R}}$. Let $\left\langle\mathcal{A}_{\alpha}: \alpha \in \Theta\right\rangle$ be a $\diamond_{\mathbb{R}^{-}}^{\prime}$-sequence. So,

(1) $\mathcal{A}_{\alpha} \subseteq \mathcal{P}(\alpha)$ for each $\alpha \in \Theta$;

(2) for every $\alpha \in \Theta$ there is a surjection of $\mathbb{R}$ onto $\mathcal{A}_{\alpha}$;

(3) for every $A \subseteq \Theta$ the set $\left\{\alpha \in \Theta: A \cap \alpha \in \mathcal{A}_{\alpha}\right\}$ is stationary in $\Theta$.

Without loss of generality, we will assume that $\varnothing \in \mathcal{A}_{\alpha}$ for each $\alpha \in \Theta$. Lemma 2.1 implies there is a set $\left\{g_{\alpha, x}:\langle\alpha, x\rangle \in \Theta \times \mathbb{R}\right\}$ such that

(1) $g_{\alpha, x}: \mathbb{R} \rightarrow \mathcal{A}_{\alpha}$ for each $\alpha<\Theta$ and $x \in \mathbb{R}$;

(2) for each $\alpha \in \Theta$ there is an $x \in \mathbb{R}$ so that $g_{\alpha, x}$ is a surjection.

For each $\alpha \in \Theta$, define $f_{\alpha}: \mathbb{R} \rightarrow \mathcal{A}_{\alpha}$ by $f_{\alpha}(z)=g_{\alpha, z_{0}}\left(z_{1}\right)$, where $z_{0}(n)=z(2 n)$ and $z_{1}(n)=z(2 n+1)$ for all $n \in \omega$. Clearly, $f_{\alpha}$ is a surjection for each $\alpha \in \Theta$. Consider the sequence $F=\left\langle f_{\alpha}(z):\langle\alpha, z\rangle \in \Theta \times \mathbb{R}\right\rangle$. We will prove that $F$ is a $\searrow_{\mathbb{R}}$-sequence. Obviously, $f_{\alpha}(z) \subseteq \alpha$ for each $\alpha \in \Theta$ and $z \in \mathbb{R}$. Let $A \subseteq \Theta$. By (3) we have that $\left\{\alpha \in \Theta: A \cap \alpha \in \mathcal{A}_{\alpha}\right\}$ is stationary in $\Theta$. Hence, the set 
$X=\left\{\alpha \in \Theta:(\exists z \in \mathbb{R})\left[A \cap \alpha \in f_{\alpha}(z)\right]\right\}$ is stationary in $\Theta$. For each $z \in \mathbb{R}$, let $Y_{z}=\left\{\alpha \in \Theta: A \cap \alpha=f_{\alpha}(z)\right\}$. Since $X=\bigcup_{z \in \mathbb{R}} Y_{z}$ is stationary, Corollary 3.2 implies that there is a $z \in \mathbb{R}$ so that $Y_{z}=\left\{\alpha \in \Theta: A \cap \alpha=f_{\alpha}(z)\right\}$ is stationary.

\section{Theorem 5.2 Assume $V=\mathbf{K}(\mathbb{R})$ and AD. Then $\diamond_{\mathbb{R}}$ holds.}

Proof Assume $V=\mathbf{K}(\mathbb{R})$ and AD. We will first prove $\searrow_{\mathbb{R}}^{\prime}$. For each $\alpha \in \Theta$, let $\mathcal{A}_{\alpha}=\mathcal{P}(\alpha)$. We will show that $\left\langle\mathcal{A}_{\alpha}: \alpha \in \Theta\right\rangle$ is a $\mho_{\mathbb{R}}^{\prime}$-sequence. Clearly, for each $\alpha \in \Theta, \mathcal{A}_{\alpha} \subseteq \mathcal{P}(\alpha)$ and, by the coding lemma (see [8, Lemma 7D.5]), there is a surjection of $\mathbb{R}$ onto $\mathcal{A}_{\alpha}$. Moreover, for each $A \subseteq \Theta$ we have that $\left\{\alpha \in \Theta: A \cap \alpha \in \mathcal{A}_{\alpha}\right\}=\Theta$. Since $\Theta$ is stationary in $\Theta$, Lemma 5.1 now implies $\diamond_{\mathbb{R}}$

The proof of Theorem 5.2 also shows, within $\mathbf{K}(\mathbb{R})$, that AD (trivially) implies $\diamond_{\mathbb{R}}^{*}$ and $\diamond_{\mathbb{R}}^{+}$. The principle $\diamond_{\mathbb{R}}^{+}$is a generalization of $\diamond_{\mathbb{R}}^{*}$ (and Jensen's principle $\diamond^{+}$) which states: there is a sequence $\left\langle\mathcal{A}_{\alpha}: \alpha \in \Theta\right\rangle$ such that

(1) $\mathcal{A}_{\alpha} \subseteq \mathcal{P}(\alpha)$ for each $\alpha \in \Theta$;

(2) for every $\alpha \in \Theta$ there is a surjection of $\mathbb{R}$ onto $\mathcal{A}_{\alpha}$;

(3) for every $A \subseteq \Theta$ there is closed unbounded $D \subseteq \Theta$ such that $A \cap \alpha \in \mathcal{A}_{\alpha}$ and $D \cap \alpha \in \mathcal{A}_{\alpha}$ for all $\alpha \in D$.

Our proof of Theorem 5.2 relies heavily on the definition of $\Theta$ and on the axiom of determinacy. Thus, it cannot be generalized to provide an alternate proof concerning our results on cardinals greater than $\Theta$. For example, the above proof cannot be adapted to prove Theorem 4.7. Furthermore, the forcing argument used to prove Theorem 4.2 shows that $\mathbf{K}(\mathbb{R}) \models \searrow_{\mathbb{R}}$ whether or not AD holds in $\mathbf{K}(\mathbb{R})$. Perhaps such a forcing argument can be used to show that other principles, which hold in $\mathbf{K}$, have analogues in $\mathbf{K}(\mathbb{R})$.

\section{References}

[1] Barwise, J., Admissible Sets and Structures: An Approach to Definability Theory, vol. 7 of Perspectives in Mathematical Logic, Springer, Berlin, 1975. Zbl 0316.02047. MR 0424560. 398

[2] Cunningham, D. W., "A covering lemma for $K(\mathbb{R})$, , Archive for Mathematical Logic, vol. 46 (2007), pp. 197-221. Zbl 1110.03044. MR 2306176. DOI 10.1007/ s00153-007-0040-8. 399

[3] Devlin, K. J., Constructibility, vol. 6 of Perspectives in Mathematical Logic, Springer, Berlin, 1984. Zbl 0542.03029. MR 0750828. DOI 10.1007/978-3-662-21723-8. 397, 403

[4] Hauser, K., "Generic relativizations of fine structure," Archive for Mathematical Logic, vol. 39 (2000), pp. 227-51. Zbl 0946.03062. MR 1758628. DOI 10.1007/ s001530050145. 397, 399

[5] Jensen, R. B., "The fine structure of the constructible hierarchy," Annals of Mathematical Logic, vol. 4 (1972), pp. 229-308. Zbl 0257.02035. MR 0309729. DOI 10.1016/ 0003-4843(72)90001-0. 397

[6] Kanamori, A., The Higher Infinite: Large Cardinals in Set Theory from Their Beginnings, Perspectives in Mathematical Logic, Springer, Berlin, 1994. Zbl 0813.03034. MR 1321144. 400 
[7] Kunen, K., Set Theory: An Introduction to Independence Proofs, vol. 102 of Studies in Logic and the Foundations of Mathematics, North-Holland, Amsterdam, 1980. Zbl 0443.03021. MR 0597342. 398

[8] Moschovakis, Y. N., Descriptive Set Theory, 2nd edition, vol. 155 of Mathematical Surveys and Monographs, American Mathematical Society, Providence, 2009. Zbl 1172.03026. MR 2526093. DOI 10.1090/surv/155. 405, 406

[9] Schimmerling, E., "Combinatorial principles in the core model for one Woodin cardinal," Annals of Pure and Applied Logic, vol. 74 (1995), pp. 153-201. Zbl 0834.03018. MR 1342358. DOI 10.1016/0168-0072(94)00036-3. 397

[10] Steel, J. R., "Scales in K(R)," pp. 176-208 in Games, Scales, and Suslin Cardinals: The Cabal Seminar, Vol. I, vol. 31 of Lecture Notes in Logic, Association for Symbolic Logic, Chicago, 2008. Zbl 1167.03032. MR 2463615. DOI 10.1017/CBO9780511546488.011. 398

[11] Steel, J. R., "An outline of inner model theory," pp. 1595-1684 in Handbook of Set Theory, Vols. 1, 2, 3, edited by M. Foreman et al., Springer, Dordrecht, 2010. Zbl 1198.03070. MR 2768698. 397

Mathematics Department

SUNY Buffalo State

Buffalo, New York

USA

cunnindw@math.buffalostate.edu

http://math.buffalostate.edu/ cunnindw/ 